\title{
Lessons from the NSW laboratory response to pandemic (H1N1) 2009 influenza
}

\author{
Sheena Adamson ${ }^{\mathrm{A}, \mathrm{D}}$, Jan Fizzell ${ }^{\mathrm{A}}$, \\ Dominic E. Dwyer ${ }^{\mathrm{B}}$, William Rawlinson ${ }^{\mathrm{C}}$ \\ and Paul K. Armstrong ${ }^{\mathrm{A}}$

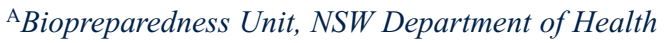 \\ ${ }^{\mathrm{B}}$ Institute of Clinical Pathology and Medical Research, \\ Westmead Hospital \\ ${ }^{\mathrm{C}}$ South Eastern Area Laboratory Services, \\ Prince of Wales Hospital \\ DCorresponding author.Email: sheena.adamson@ \\ doh.health.nsw.gov.au
}

\begin{abstract}
Procedures undertaken by NSW Health in the laboratory response to pandemic (H1N1) 2009 influenza were reviewed to assist planning for a future infectious disease emergency. Laboratory plans developed prior to the pandemic sought rapid detection of pandemic virus in the early phases of the pandemic response until demonstration of widespread community transmission, followed then by limited testing when indicated clinically. As planned, the laboratory response was based on the Pandemic Influenza Management System with a web-based outbreak management tool, NetEpi, as the single public health database used for cases and test results. This was found to be effective for tracking specimens and results, and enhanced the public health response. The sustained large volume of community testing during this mild pandemic had an adverse effect on the timely delivery of priority results.
\end{abstract}

This report provides an overview of the planning for a laboratory response in an influenza pandemic, the laboratory response to pandemic (H1N1) 2009 influenza in New South Wales (NSW), and the lessons to inform a future response to a large-scale infectious disease emergency.

\section{Pre-pandemic planning}

At the onset of the pandemic, national and NSW pandemic influenza plans were in place or in the final stages of development. ${ }^{1,2}$ In these plans, the operations for the laboratory response were determined by the phase of the pandemic. In the early phases (DELAY and early CONTAIN), the goal was the rapid detection of the pandemic virus using molecular testing of respiratory specimens, almost universally realtime polymerase chain reaction (PCR), to delay community spread by permitting the timely isolation of cases and contact tracing. After widespread community transmission had been established, testing in the later phases would then be limited to assisting clinical management, detecting new outbreaks and monitoring changes in virus strains.

\section{Managing the response}

The planned laboratory response in NSW was based on the laboratory Pandemic Influenza Management System (PIMS), together with a web-based outbreak management tool, NetEpi, as the single database for cases and near realtime test results to inform the public health response. ${ }^{3}$ NetEpi provides universal access to real-time results by authorised public health, laboratory and clinical staff irrespective of a laboratory's information management system (LIMS).

The nine steps of the PIMS (Box 1) address potential areas where delays may occur in the laboratory response, from requesting a test and specimen collection through to a reported result. These delays mainly occur prior to testing in the laboratory during processes such as specimen transport and receipt and later during the reporting of the test result. Testing in the laboratory is not usually associated with delays as this is commonly automated.

\section{Prioritising testing}

The management of suspected pandemic cases in NSW, including the collection of respiratory specimens for detection of the influenza virus, was planned to occur in emergency departments (EDs) and flu clinics, and not in general practice. This sought to ensure a standardised approach to requesting testing and the collection of specimens, and also to facilitate the rapid availability of test results. In the early pandemic phases, requests for influenza testing and the collection of respiratory specimens were to be authorised by public health or designated clinicians prior to dispatch, and a dedicated web-based request form was to be used to show the current case definition, tests and specimens required. 
Box 1. The nine steps of the laboratory Pandemic Influenza Management System in NSW

\section{Pre-laboratory \\ Step 1 Testing authorisation by public health, case entry into NetEpi $\rightarrow$ unique case number assigned}

Step 2 Specimen collection, \pm standard web-based request form

Step 3 Specimen packaging and dispatch

Step 4 Specimen transport

Step 5 Specimen tracking

\section{Laboratory}

Step 6 Specimen receipt, triage, case entry into laboratory information management system (LIMS), specimen receipt recorded in NetEpi

Step 7 Testing, result authorisation, result entry into LIMS and NetEpi

Step 8 Report result to public health and requesting clinician

Step 9 Laboratory communication: public health/laboratory liaison; situation reports; information to clinicians
In NSW, capability and capacity for initial molecular testing of respiratory specimens for an influenza pandemic virus were to be focused on two public reference laboratories in Sydney, with additional capability in other public laboratories to become subsequently serially involved. There was to be no testing in the private sector. An audit of laboratory preparedness had been completed in 2008 .

\section{Methods}

Procedures undertaken by NSW Health in the laboratory response to pandemic (H1N1) 2009 influenza were reviewed. Debriefing meetings with participating laboratories and other key stakeholders, including the NSW public health units, provided useful information about the response.

\section{Results}

In the DELAY phase of the response, respiratory specimen collection for influenza testing aimed at detecting imported cases was mainly carried out in EDs and flu clinics. In the CONTAIN phase, general practioners (GPs) were then encouraged to collect specimens for influenza testing for patients outside the pandemic influenza case definition to increase the sensitivity for detecting community transmission. Once widespread community transmission was established, Australia moved to the PROTECT phase, with testing for influenza (including the co-circulating influenza A/H3 strain of influenza) targeted at hospitalised patients, and community testing was discouraged within NSW (except where this was indicated for the clinical management of individual patients).

In the DELAY phase, nucleic acid testing (NAT) was performed solely in the two public reference laboratories using rapidly developed novel tests. These were evaluated, using control material from the World Health Organization (WHO) Collaborating Centre for Reference and Research on Influenza, for specimen types, and against current point-of-care and immunofluorescence assays for influenza. In the CONTAIN and PROTECT phases, as test numbers increased, six other public laboratories serially joined the response using commercial assays. Large-scale rapid antigen testing was also performed in the private sector in the PROTECT phase. High-volume NAT for a concurrent pertussis outbreak adversely impacted the capacity of some laboratories.

Between May-September 2009, 27060 NAT for influenza A and B were performed, of which 4230 (16\%) were positive for pandemic (H1N1) 2009 influenza virus. The peak of the pandemic occurred in NSW in the first week in July; of 10208 NAT for influenza A performed between 19 June and 24 July 2858 (56\%) were positive for pandemic (H1N1) 2009 influenza virus.

A serology (haemagglutination inhibition) test specific for pandemic (H1N1) 2009 influenza virus was developed during the PROTECT phase. It was found useful for retrospective diagnosis for patients (particularly those in intensive care) for whom respiratory specimens had not been collected, were collected too late, were collected from an inappropriate site (e.g., from the upper rather than lower respiratory tract), or in whom the diagnosis had not been made using NAT. It was also used for seroepidemiological studies.

Nearly all elements of the PIMS were used. During the DELAY and CONTAIN phases, all cases and their test results were manually entered into NetEpi, providing a universal source of data for tracking specimens and the results of testing irrespective of the laboratory, by direct access for authorised users, or general access through a NSW Health results enquiry call centre.

\section{Discussion}

The laboratory response to an infectious disease emergency must deliver appropriate and timely test results to inform public health and clinical management. Testing should be targeted to particular groups in order to deliver timely results for the intended management purpose and conserve resources; targeting will also vary according to the severity and scale of the disease. The current 2009 pandemic has been characterised by mild disease in most 
people, and by a large and sustained volume of testing by GPs, particularly in later phases, rather than the planned focus of testing in the public health sector. While testing of public health authorised specimens in early phases delivered timely results, in late CONTAIN and PROTECT phases the laboratories became overwhelmed by community requests not required for public health or clinical management.

While NetEpi provided a useful single source of case data and real-time test results, data entry and reporting by laboratories need to be automated to minimise labour and improve timeliness, and wider access to results given to clinicians and pathology services.

Adequate laboratory capability and capacity for an infectious disease emergency may be provided solely by a limited number of large reference sites, or in conjunction with multiple smaller sites. In this 2009 pandemic, NAT was performed initially in two reference laboratories, and then expanded to six other public laboratories. The advantages of the limited reference sites included expertise in rapid novel test development, close liaison during development of a test with limited quality assurance, provision of cost-effective large scale equipment, and surge staff working extended shifts. Disadvantages included nonroutine specimen transport, and lack of real-time result reporting from their LIMS to new clinicians, although this would be negated by wider real-time access to NetEpi results.

The smaller laboratories provided a more rapid local service, but had limited capacity to develop novel tests, and needed already validated tests. Some were also adversely affected by the need to use common equipment and staff for urgent testing for other pathogens.

\section{Conclusions}

The PIMS and NetEpi were effective for tracking specimens and their results. Use of these enhanced the public health response, but would be improved by automated data entry and result reporting, with wider access to real-time results for clinicians and pathology services. The large and sustained volume of community testing, which continued into the PROTECT phase despite being discouraged, had a major impact on timely delivery of results. Future plans for the laboratory response to an infectious disease emergency need to consider targeted testing in the public and private health sectors.

\section{Acknowledgments}

The enormous effort of the staff in the participating public health laboratories (Institute of Clinical Pathology and Medical Research, South Eastern Area Laboratory Services, Sydney South West Pathology Service, Pacific Laboratory Medicine Services, St Vincent's Hospital Pathology, Royal Prince Alfred Hospital Microbiology, Hunter Area Pathology Service, Nepean and Blue Mountains Pathology Service) in the laboratory response to the influenza pandemic is recognised. The contribution by the private pathology services, the NSW area health service public health units, the NSW Biopreparedness Network, and the NSW Public Health Emergency Operations Centre, is also gratefully acknowledged.

\section{References}

1. Australian Health Management Plan for Pandemic Influenza. Commonwealth of Australia, 2008.

2. NSW Health Interim Influenza Pandemic Action Plan. NSW Department of Health, 2005.

3. NSW Health. Centre for Epidemiology and Research. NetEpi. Available at: http://netepi.sourceforge.net/ (Accessed Dec 2009.) 